\title{
3D pattern of brain abnormalities in Fragile $X$ syndrome visualized using tensor-based morphometry
}

\author{
Agatha D. Lee, ${ }^{\mathrm{a}}$ Alex D. Leow, ${ }^{\mathrm{a}, \mathrm{b}}$ Allen Lu, ${ }^{\mathrm{a}}$ Allan L. Reiss, ${ }^{\mathrm{c}}$ Scott Hall, ${ }^{\mathrm{c}}$ Ming-Chang Chiang, \\ Arthur W. Toga, ${ }^{\mathrm{a}}$ and Paul M. Thompson ${ }^{\mathrm{a}, *}$ \\ ${ }^{a}$ Laboratory of Neuro Imaging, Department of Neurology, UCLA School of Medicine, 635 Charles E. Young Drive South, Suite 225E, \\ Los Angeles, CA 90095-7332, USA \\ ' Semel Institute for Neuroscience, UCLA School of Medicine, Los Angeles, CA, USA \\ ${ }^{\mathrm{c}}$ Center for Interdisciplinary Brain Sciences Research and Department of Psychiatry and Behavioral Sciences, Stanford University School of Medicine, \\ Stanford, CA, USA
}

Received 14 April 2006; revised 22 August 2006; accepted 21 September 2006

Available online 8 December 2006

Fragile X syndrome (FraX), a genetic neurodevelopmental disorder, results in impaired cognition with particular deficits in executive function and visuo-spatial skills. Here we report the first detailed 3D maps of the effects of the Fragile $X$ mutation on brain structure, using tensor-based morphometry. TBM visualizes structural brain deficits automatically, without time-consuming specification of regions-ofinterest. We compared 36 subjects with FraX (age: $14.66 \pm 1.58$ S.D., 18 females/18 males), and 33 age-matched healthy controls (age: $14.67 \pm$ 2.2 S.D., 17 females/16 males), using high-dimensional elastic image registration. All 69 subjects' 3D T1-weighted brain MRIs were spatially deformed to match a high-resolution single-subject average MRI scan in ICBM space, whose geometry was optimized to produce a minimal deformation target. Maps of the local Jacobian determinant (expansion factor) were computed from the deformation fields. Statistical maps showed increased caudate $(10 \%$ higher; $p=0.001)$ and lateral ventricle volumes (19\% higher; $p=0.003)$, and trend-level parietal and temporal white matter excesses $(10 \%$ higher locally; $p=0.04)$. In affected females, volume abnormalities correlated with reduction in systemically measured levels of the Fragile $X$ mental retardation protein (FMRP; Spearman's $r<-0.5$ locally). Decreased FMRP correlated with ventricular expansion $(p=0.042$; permutation test $)$, and anterior cingulate tissue reductions $(p=0.0026$; permutation test $)$ supporting theories that FMRP is required for normal dendritic pruning in fronto-striatal-limbic pathways. No sex differences were found; findings were confirmed using traditional volumetric measures in regions of interest. Deficit patterns were replicated by performing statistics after logarithmic transformation, which may be more appropriate for tensor-valued data. Investigation of how these anomalies emerge over time will accelerate our understanding of FraX and its treatment.

(C) 2006 Elsevier Inc. All rights reserved.

\footnotetext{
* Corresponding author. Fax: +1 3102065518.

E-mail address: thompson@loni.ucla.edu (P.M. Thompson).

Available online on ScienceDirect (www.sciencedirect.com).
}

\section{Introduction}

Fragile X syndrome (FraX) is the commonest inherited form of mental disability, occurring in approximately 1 in 2000-6000 live births (Gustavson et al., 1986; De Vries et al., 1997). FraX results from a specific single gene mutation that alters the course of brain development and cognition throughout life. As expected with an Xlinked disorder, affected women, who carry two X chromosomes, are somewhat protected from the full impact of the syndrome - they typically exhibit normal cognitive function or only relatively mild mental retardation, with difficulties in socialization, anxiety, and moderate learning and attention deficits. Men, who rely on a single $\mathrm{X}$ chromosome, may be moderately to severely mentally retarded, with particular deficits in executive function, visuo-spatial skills, and visuo-motor coordination. IQ often declines during childhood in affected males (Hodapp et al., 1990) and measures of cognitive performance fail to show the normal correlations with volumetric brain measures (Eliez et al., 2001).

The neurobehavioral profile of FraX overlaps with autism, a phenomenologically defined condition. Unlike idiopathic autism however, the cause of FraX is known: an abnormally expanded number of trinucleotide repeats (with genetic sequence CGG) in the initial (5) untranslated region (UTR) of the Fragile X mental retardation gene' (FMRI). In individuals with more than 200 of these CGG repeats, hypermethylation occurs in promoter region of this gene. This reduces or completely silences the transcription and translation of a protein known as the FMRI protein (FMRP), which is required for healthy brain development. FMRP is normally expressed in neurons throughout the brain, with particularly high expression in the cerebellum, hippocampus and nucleus basalis (Tamanini et al., 1996).

Downstream effects of reduced FMRP in FraX include altered arborization of cortical dendrites and gray matter enlargement (Irwin et al., 2000; Willemsen et al., 2004; Beckel-Mitchener and Greenough, 2004; Galvez and Greenough, 2005). Not all gray matter structures are affected to the same degree. Caudate volumes are on average $28 \%$ 
larger in affected males and 13\% larger in affected females with the full mutation (Eliez et al., 2001). Some investigators (Reiss et al., 1994) but not others (Jakala et al., 1997) found enlarged hippocampi in affected males, and enlarged thalamic volume in affected females (Eliez et al., 2001), but there are currently no detailed 3D maps of the profile of abnormalities in the illness.

Eliez et al. (2001) compared 35 adolescents with DNAconfirmed FraX (aged 4-19 years, mean age: 10.2 \pm 3.8 years) with 85 typically developing children. The normal age-related decline in cortical gray matter was reduced in affected males, adding to evidence that FMRP is required for normal dendritic and synaptic pruning. Gray matter volumes may remain abnormally high if pruning is reduced or delayed. Histologic studies of FraX have also found decreased synaptic pruning and immature dendritic morphology (Hinton et al., 2005; Wisniewski et al., 1991) or excessively long and numerous dendrites in the cortex (Weiler and Greenough, 1993; Galvez and Greenough, 2005).

If dendritic and synaptic pruning are abnormal in FraX, it would help to understand whether these disturbances are widespread or localized in the brain. Computational anatomy techniques can visualize the $3 \mathrm{D}$ pattern of structural anomalies revealing which structures show selective deficits. Our recent dynamically changing maps ('time lapse movies') of cortical maturation have visualized in vivo - the trajectory of cortical myelination and dendritic pruning seen in post mortem studies, based on living subjects scanned serially with MRI (Sowell et al., 2003, 2004; Gogtay et al., 2004; see Toga et al., 2006 for a detailed comparison).

Here we used a technique called tensor-based morphometry (TBM; see Ashburner and Friston, 1999; Davatzikos et al., 1996, 2001; Thompson et al., 2000; Chung et al., 2001, 2003, 2004; Fox et al., 2001; Shen and Davatzikos, 2003; Studholme et al., 2001, 2003, 2004 and Teipel et al., 2004, for related work) to establish the $3 \mathrm{D}$ profile of structural abnormalities in FraX. We correlated abnormalities with measured levels of FMRP, the reduction of which results in the illness. TBM is automated and avoids time-consuming specification of regions of interest typically required in traditional volumetric studies. Traditional morphometric studies, which assess the volume of a specific set of structures, are generally laborintensive and cannot visualize the profile of deficits at the voxel level throughout the brain.

We tested four hypotheses: (1) ventricular CSF expansion and gray matter enlargement would be detected in both males and females with FraX; (2) the degree of gray matter enlargement would be greater in males, who suffer the full impact of the Xlinked mutation; (3) greater gray matter enlargement and ventricular expansion would correlate with the degree of reduction in FMRP protein levels measured systemically; and (4) this correlation would be greater in females, who are heterozygous for the mutation and therefore exhibit greater variation in FMRP levels. Finally, we compared our TBM results with conventional morphometric analysis using regions of interest. We expected our 3D maps to provide greater statistical power to associate brain structure abnormalities with diagnosis, sex, and FMRP levels, as well as providing new biological measures of disease burden.

\section{Methods}

\section{Subjects and MRI scanning}

Participants included 36 subjects with FraX (mean age 14.66, S.D. $=1.58,18$ females and 18 males), and 33 age-matched healthy controls (mean age 14.67, S.D. $=2.2$, 17 females and 16 males). FraX subjects were recruited from throughout the U.S.; their diagnosis was confirmed by DNA analysis. Standardized Southern blotting and polymerase chain reaction (PCR) analyses were performed, followed by FMR1-specific probe hybridization (Schapiro et al., 1995). FMRP levels were determined by immunostaining techniques (Willemsen et al., 2004), which revealed the percentage of peripheral lymphocytes containing FMRP. This measure of protein levels was used as a covariate of interest in the image analysis (described later). All subjects were scanned on a 1.5 T GE Signa scanner with a high-resolution T1weighted spoiled gradient recalled (SPGR) 3D MRI sequence. The following scanning parameters were used: $\mathrm{TR}=35 \mathrm{~ms}, \mathrm{TE}=6 \mathrm{~ms}$, flip angle $=45^{\circ} ; 24 \mathrm{~cm}$ field of view; 124 slices in the coronal plane; $256 \times 192$ matrix; acquired resolution $=1.5 \times 0.9 \times 1.2 \mathrm{~mm}$.

\section{Image analysis}

\section{Overview of TBM and image deformation approach}

TBM evaluates regional differences in the volumes of brain substructures by globally aligning all brain images to a common brain template, before applying localized deformations to adjust each subject's anatomy to match the brain template in detail. The steps are summarized in Fig. 1. Briefly, all images are nonlinearly deformed to match a preselected brain template. Then, the Jacobian determinant (i.e., the local expansion factor) of the deformation fields is used to gauge the local volume differences between the individual images and the template. These are analyzed statistically to identify group differences in brain structure, such as localized atrophy or tissue excess.

\section{Image pre-processing}

First, extra-cerebral tissues (e.g., scalp, meninges, brain stem) were deleted from the images. To do this, we created a binary intracranial mask of the cerebrum using BSE (Shattuck and Leahy, 2002), a brain surface extraction algorithm that combines 3D image filtering, edge detection, and morphological processing. Additional manual editing was used to fix any errors and completely separate cerebral from non-cerebral tissues such as scalp and dura. For a more detailed analysis of cerebellar regions, we isolated the cerebellum manually from each image, using careful anatomic criteria to consistently separate the cerebellum from the brain stem, guided by the Schmahmann Atlas of the Human Cerebellum (Schmahmann et al., 2000); we followed the cerebellar cortical contour where the boundary between the cerebellum and brainstem was not distinct. We then used the same methods presented for the cerebrum to generate a specific minimum deformation template (MDT) for the cerebellum, which was then used as a target for performing elastic nonlinear registration. The benefit of doing this is that the cost function that optimizes the alignment of anatomy across subjects was based entirely on the data in the cerebellar region.

The MRI brain scan of each subject, and its brain mask, were coregistered with scaling (9-parameter transformation) to a highresolution single-subject average MRI brain scan in ICBM space (the Colin27 brain template; Holmes et al., 1998: downloadable at http:// www.loni.ucla.edu/Atlases/Atlas_Detail.jsp?atlas_id=5). This step adjusts for individual differences in global brain scale and head alignment (the amount of scaling was retained, and used as a covariate in subsequent analyses). As in other TBM studies (e.g., Studholme et al., 2001; Davatzikos et al., 2001), we preferred registration to a single subject's image versus a multi-subject average intensity atlas as it had 


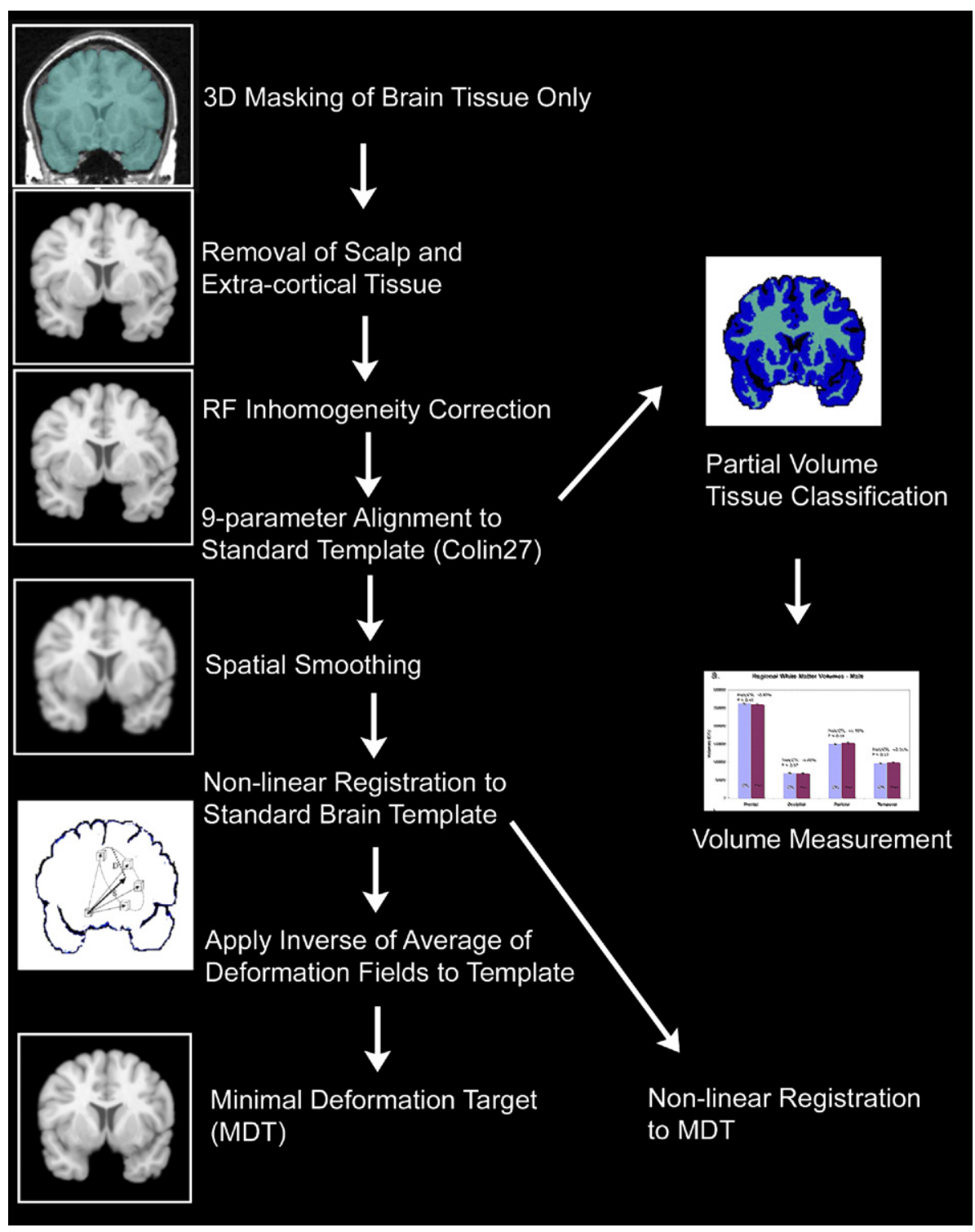

Fig. 1. Analysis sequence. This schematic shows the steps used to analyze the images. Images were digitally edited to remove extracerebral tissue, intensitycorrected, and globally aligned to a standard space. Tissue classification was performed to quantify gray and white matter volumes. To avoid bias in aligning anatomy, all subjects' images were first nonlinearly aligned to a high-resolution single subject brain template (Colin27; Holmes et al., 1998). The average deformation required to do this was expressed as an inverse transformation in the coordinate system of the target brain template, and the brain template was resampled using this transform so that its geometry reflected the mean shape of the images in the study (see Kochunov et al., 2001). Images were then re-registered to the resulting "minimal deformation target" (MDT). The profiles of expansions and contractions were analyzed statistically to investigate volumetric differences in anatomy.

higher contrast, better spatial resolution and sharper features; template optimization for TBM is the subject of further on-going study by us and others (Kochunov et al., 2002, 2005; Studholme and Cardenas, 2004; Twining et al., 2005).

After global registration of each image, the non-parametric nonuniform intensity normalization method (N3; Sled et al., 1998) was applied to each scan to correct for intensity inhomogeneities due to radiofrequency $(\mathrm{RF})$ field non-uniformities. This iterative approach estimates the multiplicative bias field to optimize the sharpness of the tissue intensity histogram. For each scan, 200 iterations of N3 correction (Montreal Neurological Institute: nu_correct, version 1.02) were applied using the corresponding individual's brain mask as the region of interest. To assist subsequent nonlinear registration and to increase signal-to-noise ratio, images were digitally filtered with an isotropic 3D Gaussian kernel, with a full-width-halfmaximum of $1.5 \mathrm{~mm}$.

\section{Generating a minimal deformation target (MDT)}

Based on concepts in Kochunov et al. (2001, 2005), we generated a "minimal deformation target" (MDT) target brain image based on common features of a group of three-dimensional MR brain images. In past studies, registering data to an image with the group mean geometry has been shown to (1) reduce the deformation required to align each individual's image (thereby making image registration more robust and accurate, by avoiding non-global minima of the cost function), and (2) reduce the bias in 
statistical analyses by expressing results in a mean spatial coordinate system (Woods, 2003; Leow et al., 2006, submitted for publication). [Note that the "average" anatomy may also be defined as the image that requires least deformation energy to deform onto all the others, rather than least distance-each formulation of this energy produces a slightly different mean image; Miller, 2004; Avants and Gee, 2004.]

To create the MDT, each individual in the study was nonlinearly registered to the Colin 27 template, by computing an inverse-consistent 3D elastic deformation vector field to deform one $3 \mathrm{D}$ image to match the other, maximizing the mutual information (MI) between the images (Leow et al., 2005). The minimal deformation target (MDT) was then generated by applying the inverse of the mean displacement field from all subjects to the Colin 27 brain (Kochunov et al., 2005). Fig. 2 shows representative axial slices from the Colin27, MDT image, and a randomly selected individual from the study.

\section{$3 D$ nonlinear registration to the MDT}

Each scan was then nonlinearly re-registered to the MDT using the same MI-based inverse-consistent elastic registration algorithm (Leow et al., 2005). Briefly, a multi-resolution scheme was used, computing deformations using Fast Fourier Transforms (FFTs) at three successively increasing spatial resolutions: $32 \times 32 \times 32$, $64 \times 64 \times 64$, and $128 \times 128 \times 128$ voxels. Numerical convergence was checked every 20 iterations, and was defined as the point at which MI failed to increase by 0.001 after the prior iteration. 300 iterations were computed at each FFT resolution before increasing the resolution by a factor of 2 in each dimension (with the time step decreased to one-tenth of its previous value).

\section{Analysis of deformation using Jacobian determinants (local expansion factors)}

The Jacobian determinant operator was applied to the resulting deformation fields to create Jacobian maps that show the local expansion factors (Jacobian $>1$ ), or contractions (Jacobian <1) required to deform a given subject's anatomy to match the template (for related work, see Fox et al., 1996; Ashburner et al., 1998; Thompson et al., 2000; Studholme et al., 2001). In the Jacobian maps, values such as 0.9 and 1.1 would indicate that specific regions are respectively $10 \%$ smaller, or $10 \%$ larger, than corresponding structures in the MDT brain. Individual subjects' Jacobian maps were color-coded and overlaid on their anatomical images to assist in confirming registration accuracy. In all analyses, Jacobian determinant values were first subjected to a $\log$ transformation because the null distribution of the log (Jacobian) is closer to Normal than that of the Jacobian determinant, which is skewed and bounded below by zero (Ashburner and Friston, 2000; Cachier and Rey, 2000; Woods, 2003; Avants et al., 2006; Arsigny et al., 2005; see Leow et al., 2006, submitted for publication, for discussions of why the Jacobians are typically logged before statistical analysis). We tested the significance of any difference between the mean $\log$ (Jacobian) of the FraX and the control groups using voxel-wise multiple regression.

\section{Tissue classification}

To further examine the volume differences identified with TBM, we performed tissue classification of the individual subjects' MRI data into gray matter, white matter and CSF, using an automated algorithm (PVC, or partial volume classifier; Shattuck et al., 2001). PVC takes into account the partial volume effect when fitting a Gaussian mixture distribution to the intensity histograms in the neighborhood of each classified image voxel. Regions of interest representing the lobes and subcortical regions (including the basal ganglia and lateral ventricles) were defined on the Colin 27 brain template and adapted to each individual subject, via the nonlinear registration fields. This allowed approximate quantitation of gray and white matter in each lobe, and the volumes of each tissue type were compared between groups.

\section{Statistical testing for group differences in tissue volumes}

Both the geometric mean and simple arithmetic means of the Jacobian determinants were used to generate statistical parametric maps of group differences in local tissue volumes. To adjust for multiple comparisons of mean logged Jacobian values inside specific regions of interest (ROIs) in the brain, permutation tests using voxel-wise $t$ tests were applied to the maps of two-sided twosample $t$ statistics. To test the null hypothesis of no difference between the two populations (FraX and controls), we resampled the observations by randomly assigning subjects to groups (of the same sample sizes as the original groupings). The percentage of voxels was computed, inside the chosen ROI, that had $T$ statistics exceeding a pre-defined, fixed threshold (here $p=0.05$ was used as the primary threshold). Corrected $p$-values for the observed group difference were then determined by counting the number of

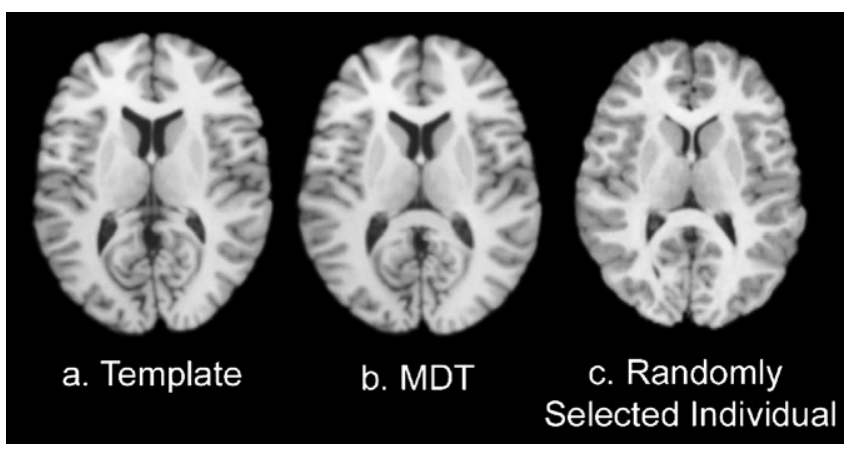

Fig. 2. Templates used for TBM. Shown here are axial slices from the Colin27 brain template (a), a specially-constructed 'minimally deformed target' image (MDT; (b); see Kochunov et al., 2001), and a randomly selected subject from the study (c). The template's overall shape in (a) has been deformed in (b) to assume the mean shape of the sample of FraX and control scans-i.e., its shape in (b) appears closer to (c) than to (a). Registering data to the MDT can reduce bias in the registrations, and in the statistical analyses, and can also make the registrations more accurate (see Kochunov et al., 2001, 2005 for our prior work on this). 
random permutations whose percentage of significant voxels, defined above, was greater than that observed before randomization of the data. 10,000 permutations were used to obtain the final corrected $p$ value.

\section{Correlation with protein levels}

In the FraX group, we also used Spearman's rank test to examine correlations between FMRP protein levels and anatomical differences (indexed by logged Jacobian values). In addition to the voxel-wise significance, correlation $(r)$ values were also mapped, to determine the proportion of variance explained by protein levels. Omnibus significance was confirmed using ROI-based permutation testing. As before, suprathreshold voxels were counted and compared to their null distribution ascertained from 10,000 random assignments of the covariates to the subjects.

\section{Results}

\section{Overall volumetric differences}

To provide context for the TBM analyses, regional tissue volumes for selected gray and white matter regions where effects were hypothesized are presented in Figs. 3 and 4. These volumes
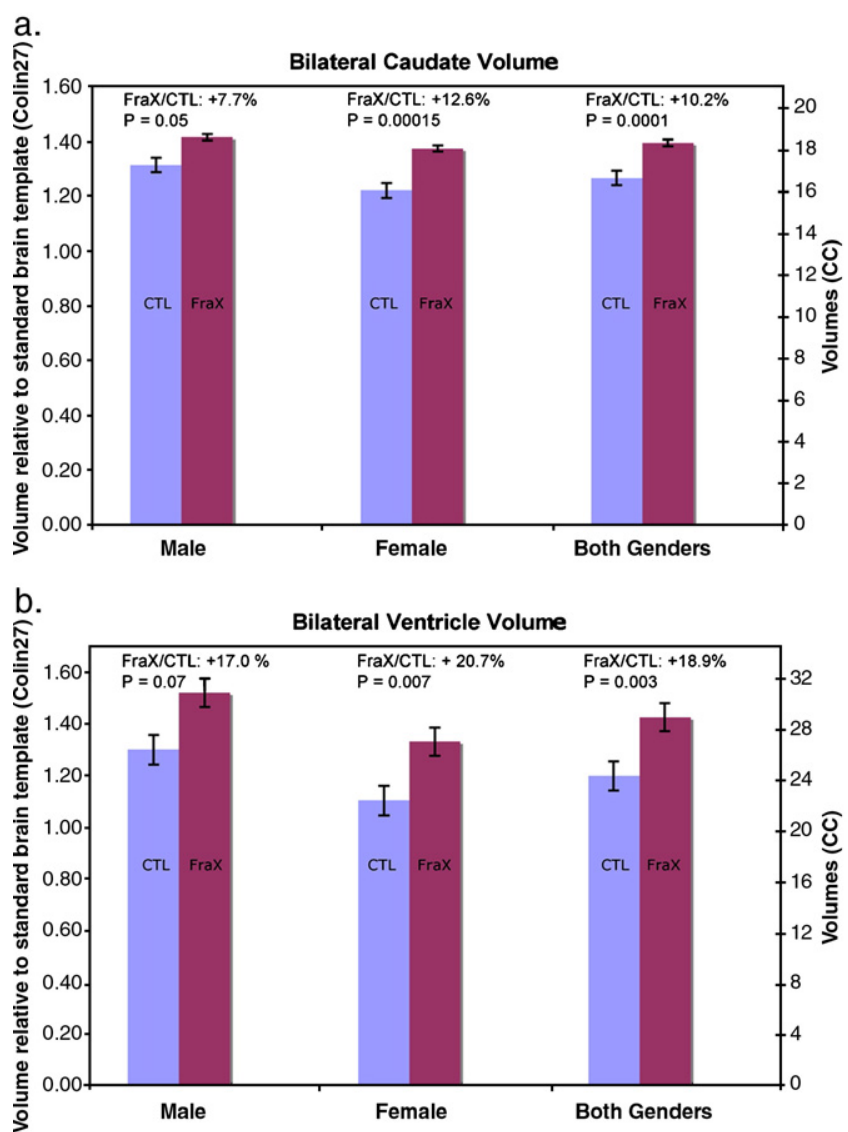

Fig. 3. Brain structure volumes are compared for Fragile $\mathrm{X}$ and healthy control subjects. Means and SE measures (error bars) are shown for the caudate (a) and lateral ventricles (b). FraX subjects show significant expansion of the caudate and ventricles. The observed differences support prior findings of increased caudate volume in FraX females (Eliez et al., 2001). CTL, Control; FraX, Fragile X syndrome. a. $\quad$ Regional White Matter Volumes - Male

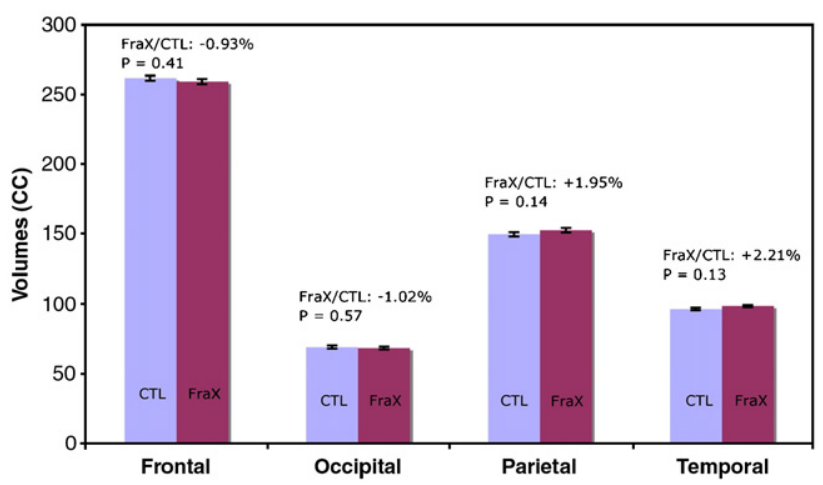

b. Regional White Matter Volumes - Female

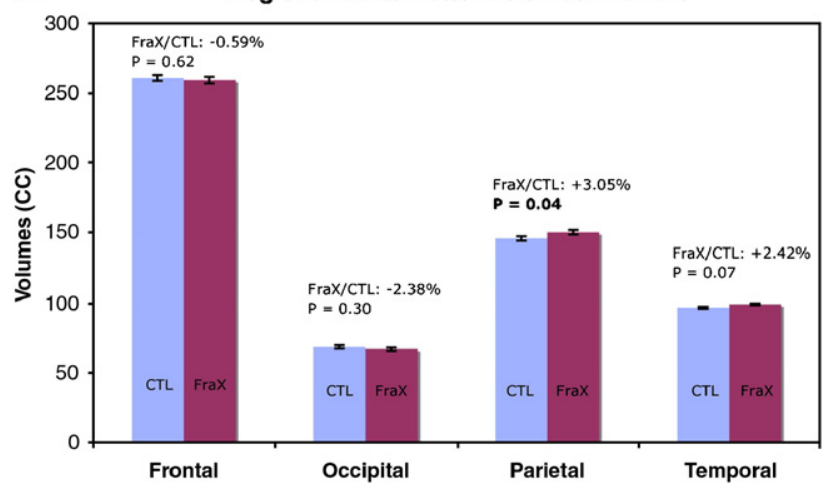

C. Regional White Matter Volumes - Male \& Female

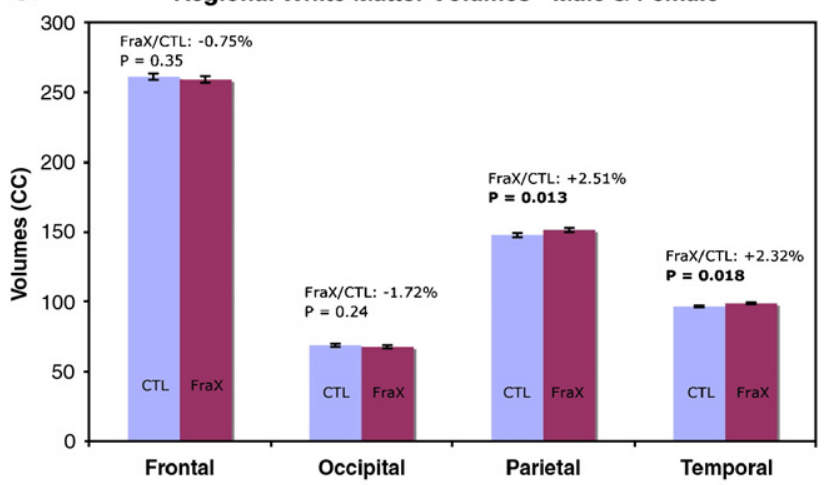

Fig. 4. Comparison of lobar white matter volumes in FraX syndrome and healthy controls. Means and S.E. measures (error bars) for the white matter volumes of each of four cerebral lobes are shown for males (a), females (b) and both genders pooled (c). After Bonferroni correction for multiple regions assessed, parietal and temporal white matter show only trend-level increases in FraX when genders are pooled.

were computed by warping labels (binary masks) pre-contoured on the Colin27 brain template onto the individual scans. All the volumes are obtained from globally adjusted scans to eliminate variance related to individual differences in brain scale. Global scaling factors were not significantly different between FraX and control groups. However, analysis of scaling factors revealed that males' cerebral volumes were $12 \%$ larger than females' $\left(p=1.7 \times 10^{-6}\right)$ for controls and $14 \%$ larger within the FraX group $\left(p=4.9 \times 10^{-5}\right.$; sex differences in brain size in normal developing children are discussed in Pfefferbaum et al., 1994; Giedd et al., 1996, 1999; Reiss et al., 1996). 


\section{Caudate and lateral ventricles}

As hypothesized, when data from both genders and both hemispheres were pooled, mean caudate volumes were $10.2 \%$ higher in the FraX group compared to controls $(p=0.0001)$. Contrary to our prediction that males would show greater abnormalities (as the syndrome is X-linked), the caudate enlargement was $12.6 \%$ in FraX females $(p=0.00015)$ but only $7.7 \%$ in FraX males $(p=0.05)$, relative to controls of the same sex.

Lateral ventricular volumes were also significantly larger in FraX compared to controls $(18.9 \%$ larger when genders were pooled; $p=0.003$ ). Due to the large normal variation in ventricular anatomy, this effect was only significant in females when the genders were analyzed separately (males: $17.0 \%$ enlargement, $p=0.07$, not significant; females: $20.7 \%$ enlargement, $p=0.007$ ).

ANOVA was also performed, using the nonparametric Friedman test, to evaluate any interactions between gender and diagnosis in explaining these volume differences. Surprisingly, no gender difference was found. In other words, the disease effect was not found to be more pronounced for FraX males than FraX females in either the caudate or lateral ventricular regions.

\section{Lobar white matter}

There was trend-level evidence for a slight excess in white matter volumes in FraX, even after adjustment for total cerebral volume was made (as noted before, total cerebral volume was not different between groups). The temporal and parietal white matter volumes were slightly elevated in FraX when the sexes were pooled (by $2.3 \%$ and $2.5 \%$ on average, $p=0.018$ and 0.013 ), but when genders were split, only the parietal white matter in females showed a disease related increase $(3.1 \% ; p=0.04)$. These findings should be interpreted as trends that are apparent only when the genders are pooled, as a Bonferroni correction should be applied to adjust for multiple comparisons.

\section{Mapping local tissue differences with TBM}

Jacobian maps (showing volumes of structures relative to the mean brain template) were averaged for FraX and control groups and are shown in Fig. 5. As sex differences were hypothesized, each diagnostic group is shown with results stratified by gender, and with genders pooled. In Fig. 5, and as confirmed by the statistical maps in Fig. 6, the lateral ventricle in FraX is abnormally expanded compared to controls and the effect is significant in both males and females considered separately (red colors indicate up to $30 \%$ expansion locally compared to the MDT). In the periventricular white matter, temporal and parietal white matter regions show more volume in FraX group compared to controls (up to $10 \%$ expansion; Fig. 7). The major white matter enlargement is concentrated subcortically in the vicinity of the ventricles. Group differences assessed using geometric means did not differ from those computed by conventional scalar averaging of the Jacobian determinants. Log-transformation did not significantly alter the effects found for the scalar Jacobian maps.

Fig. 7 shows the profile of local volumetric differences between the two groups (FraX mean divided by control mean). FraX subjects had larger volumes bilaterally for the caudate $(p=0.0001)$, and lateral ventricle volumes $(p=0.003)$. After (but not before) adjusting for individual brain volume differences, parietal and temporal white matter volumes were greater in FraX - by $2.51 \%$ $(p=0.027)$ and $2.32 \%(p=0.038)$. Fig. 8 shows variance maps for each group, based on the standard deviation of the Jacobian determinants (relative tissue volumes). Random, non-diseaserelated variability tends to reduce statistical power for detecting group differences; however, subcortical differences were detected in spite of relatively highly volume variation in the lateral ventricles. As shown in Fig. 7, and confirmed by the significance maps (Fig. 6b), the occipital region and deep frontal regions overlying the insula show evidence for reduced volumes relative to controls. The number of significant voxels is slightly higher for the

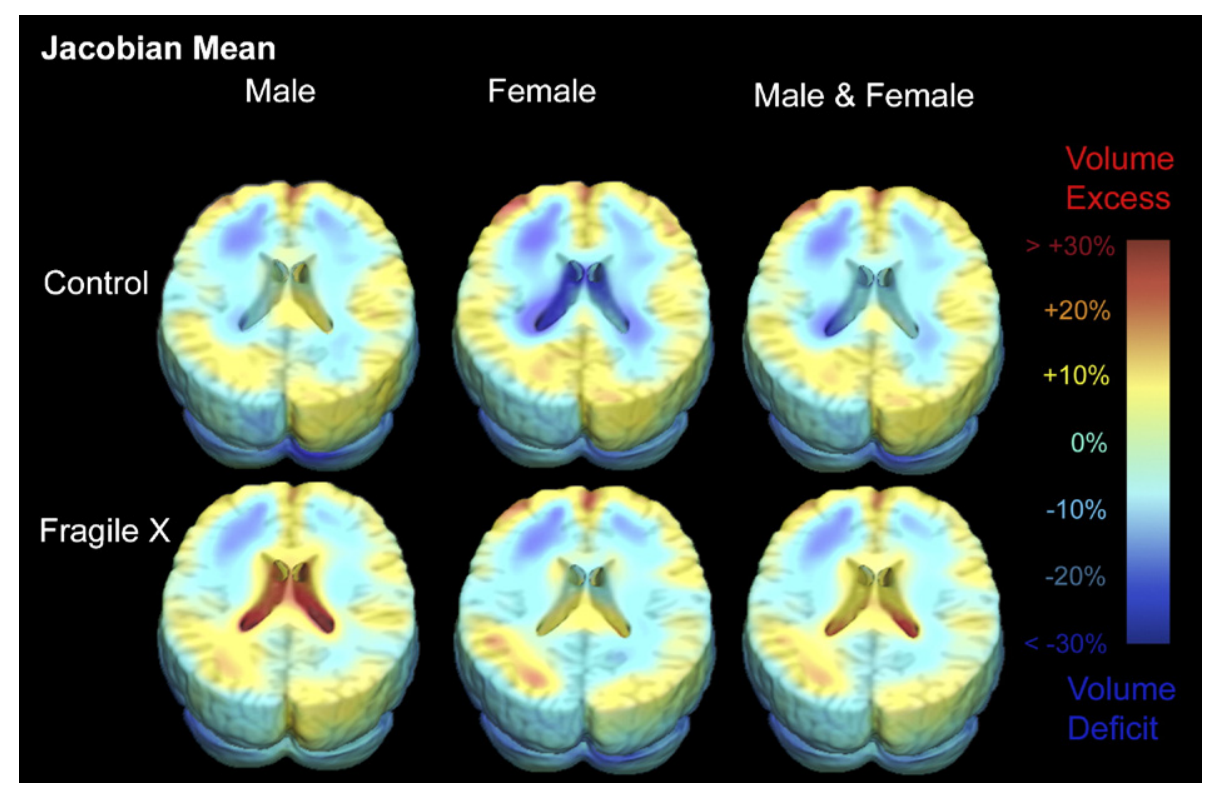

Fig. 5. Mean tissue expansion/deficit maps. The mean Jacobian maps (indicating relative tissue volumes) for control and Fragile $\mathrm{X}$ males, females and both genders pooled ( $N=33$ controls - 16 males and 17 females; 36 FraX -18 males and 18 females). The mean tissue expansion/deficit in FraX is shown as a percentage of the corresponding region's volume in the MDT. Red colors in the lateral ventricles of male FraX subjects indicate a $30 \%$ expansion, on average, relative to the corresponding region in the MDT. 


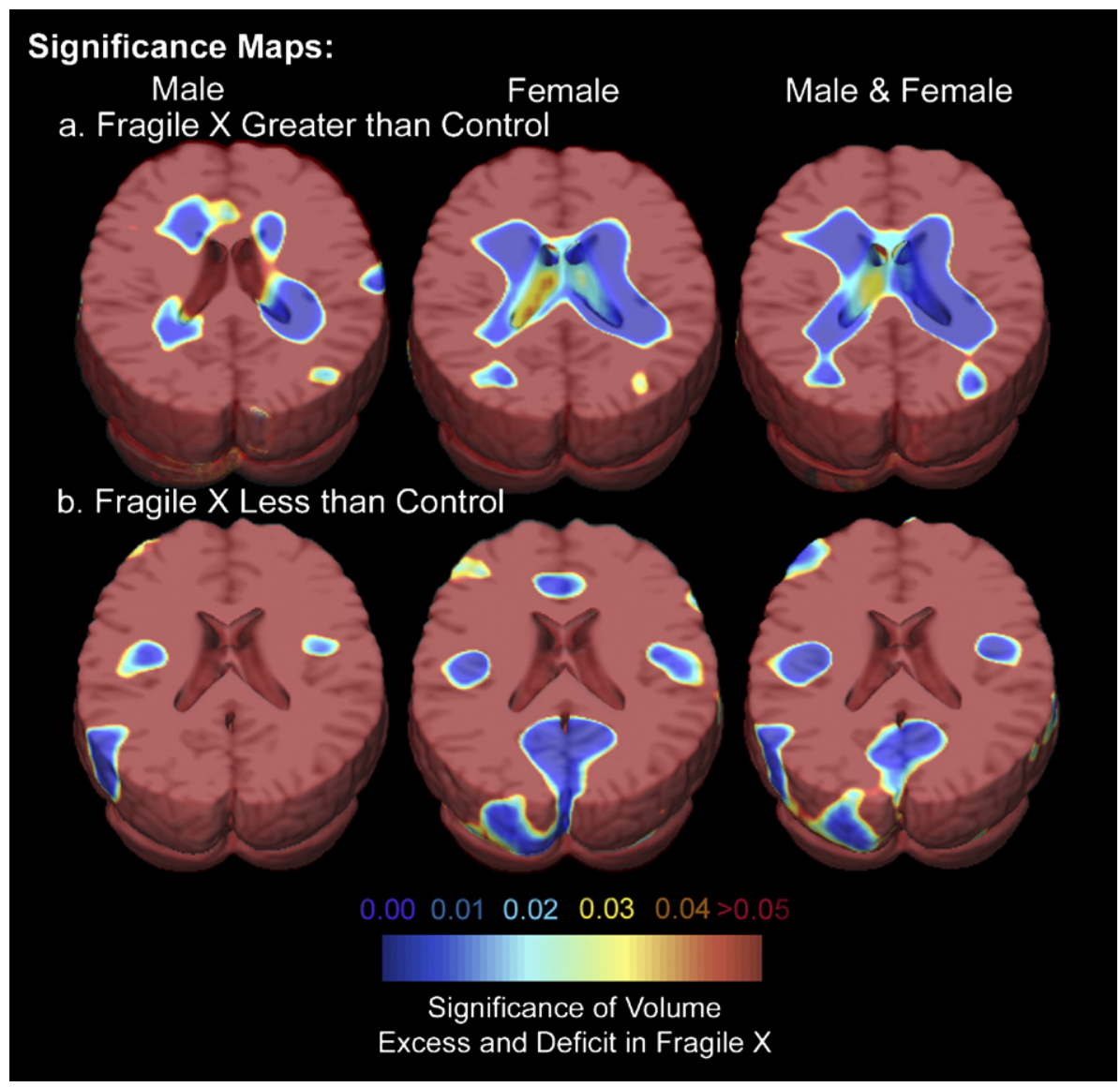

Fig. 6. Significance maps for group differences in local brain volume between FraX and controls. Significant volume differences in FraX versus healthy controls are shown for males (left panels in a and b), females (middle of $a$ and $b$ ) and for all subjects (right panels in a and $b$ ). In all maps, FraX subjects have significant excess volumes in the ventricle and caudate regions (blue colors in panel a). Greater variance in males (Fig. 8) may explain the smaller effect size in males (left panels in $\mathrm{a}$ and $\mathrm{b}$ ), although the gender $\mathrm{x}$ diagnosis interaction was not significant. (b) shows that subjects with FraX have significant volume deficits in medial occipital regions and some temporal lobe regions.

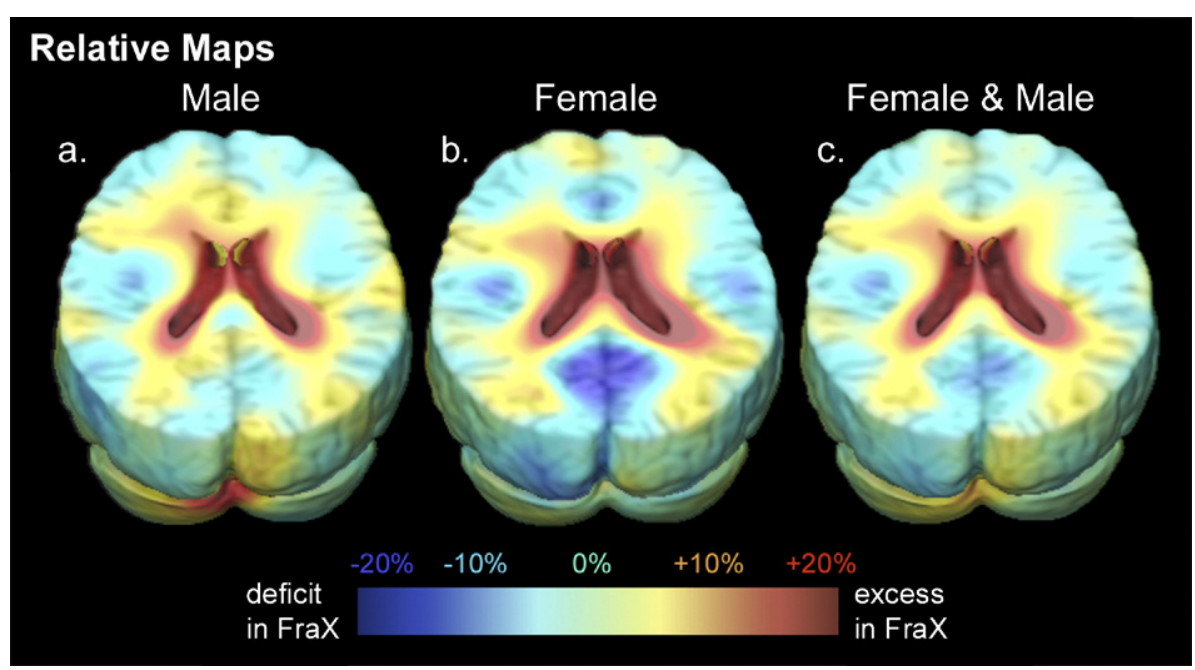

Fig. 7. Relative tissue volume maps for FraX and control groups. Jacobian maps are shown using arithmetic means for males (a), females (b) and in both genders pooled (c). In the deep white matter, basal ganglia and lateral ventricles, mean volumes are greater in FraX than normal controls. (b): medial occipital regions show volume reductions in females with FraX, relative to female controls. 


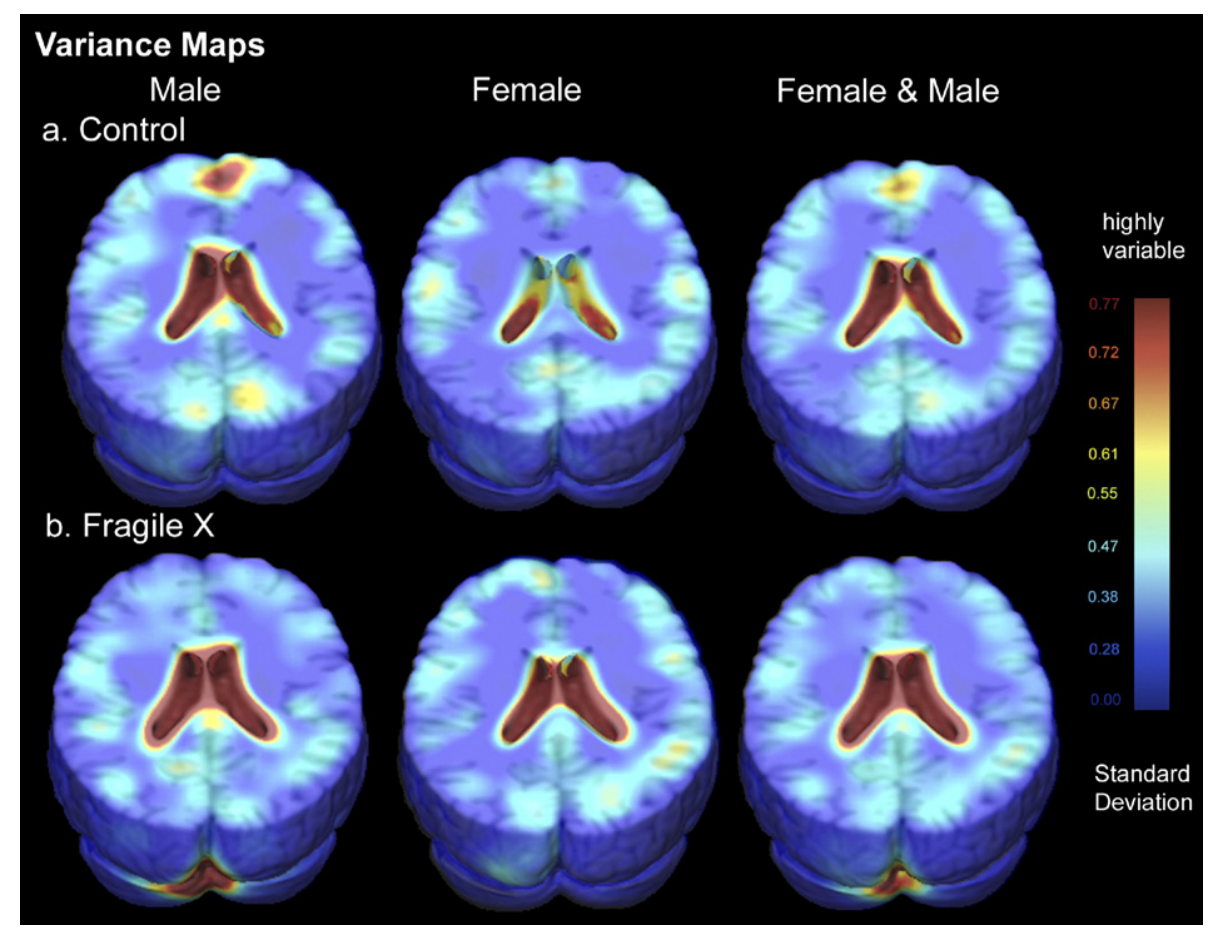

Fig. 8. Variance of tissue volumes within each group. a, controls and b, FraX. The variance maps for each group are shown, based on the standard deviation of the Jacobian determinants (relative tissue volumes). The statistical power to detect group differences is spatially variable and depends on the volumetric variability of each region across subjects. Although random, non-disease-related variability reduces statistical power for detecting group differences. Even so, subcortical differences were detected even though the volume variation in the lateral ventricles is relatively high.

comparison of female groups, but a similar pattern is seen in males and when both genders are combined. The corrected $p$-value for the deficit, using the whole brain volume as a search region, is $p=0.002$ after multiple comparisons correction, when the genders are pooled. In males with FraX, the medial occipital region is highly variable in volume (see Fig. 8), which may explain why the medial occipital deficit is seen in the females with FraX but not in the males with FraX.

Table 1 reports average values for total brain volumes, after careful editing of scalp and dura from the images, broken down into groups by diagnosis and gender: male controls, female controls, male and female controls pooled, male subjects with FraX, female subjects with FraX, and both male and female subjects with FraX.

Before global scaling, total brain volumes were significantly smaller in females than males in both FraX and controls ( $p=0.0002$ for controls, $p=0.000045$ for FraX). After global scaling, significant differences disappeared, as expected. Also as would be expected, overall scaled tissue volumes were not associated with diagnosis in either gender, and the mean difference was negligible ( $\sim 1 \%$ less volume for FraX compared to controls).

\section{Maps of the cerebellum}

Fig. 11 shows the mean local volume difference in the cerebellum between FraX and Controls (FraX mean divided by the control mean). Male subjects with FraX have statistics at the voxel level denoting smaller volumes bilaterally in the posterior cerebellar lobule than control males. Female subjects with FraX have voxel-level statistics indicating smaller cerebellar lobule V and VI volumes than control females; a posterior cerebellar lobe deficit was not detected in females. When genders were pooled, lobule $\mathrm{V}$ and VI and posterior lobar regions all showed deficits of around $5 \%$. Permutation tests on the suprathreshold volume of statistics confirm the presence, but not localization, of these effects, so these effects are confirmed as present but not definitively localized to these regions. These results were confirmed by the statistical map, which shows the significance of the group mean differences in volume (Fig. 10). Corrected $p$-values, computed via permutation testing with the whole cerebellar volume mask as a search region, are all less than 0.001, indicating deficits for FraX in groups containing males alone, females alone, and in pooled

Table 1

Means and standard deviations (S.D.) for total brain tissue volume, in cc, before and after adjustment for global brain scale: data are reported for groups of male controls, female controls, male and female controls, male FraX, female FraX, male and female FraX

\begin{tabular}{lllrlll}
\hline & & \multicolumn{2}{l}{$\begin{array}{l}\text { Before global } \\
\text { scaling }\end{array}$} & & \multicolumn{2}{l}{$\begin{array}{l}\text { After global } \\
\text { scaling }\end{array}$} \\
\cline { 3 - 4 } \cline { 7 - 8 } \cline { 7 - 8 } & & Mean & S.D. & & Mean & S.D. \\
\hline Control & Male & 1428.14 & 122.90 & & 1826.67 & 67.87 \\
& Female & 1278.97 & 81.26 & & 1839.03 & 51.05 \\
& Genders pooled & 1351.30 & 126.93 & & 1833.03 & 59.18 \\
Fragile X X & Male & 1416.79 & 101.53 & & 1798.93 & 65.60 \\
& Female & 1269.47 & 82.55 & & 1833.98 & 45.44 \\
& Genders pooled & 1340.44 & 118.07 & & 1815.96 & 58.65 \\
\hline
\end{tabular}

This measure of total brain volume includes gray matter, white matter and cerebrospinal fluid. The cerebrum and cerebellum are included in the measurement. 
Table 2

Corrected $p$-values for the group morphometric differences (FraX vs. controls) within the cerebellar region

\begin{tabular}{llllll}
\hline & \multicolumn{2}{l}{ Before global adjustment } & & \multicolumn{2}{l}{ After global adjustment } \\
\cline { 2 - 3 } \cline { 5 - 6 } & $\begin{array}{l}\text { Significant } \\
\text { excess }\end{array}$ & $\begin{array}{l}\text { Significant } \\
\text { deficit }\end{array}$ & & $\begin{array}{l}\text { Significant } \\
\text { excess }\end{array}$ & $\begin{array}{l}\text { Significant } \\
\text { deficit }\end{array}$ \\
\hline Male & 0.1043 & $\mathbf{0 . 0 0 0 1}$ & & 0.1045 & $\mathbf{0 . 0 0 0 1}$ \\
Female & $\mathbf{0 . 0 0 0 1}$ & $\mathbf{0 . 0 0 0 1}$ & & 0.1049 & $\mathbf{0 . 0 0 0 1}$ \\
Both genders pooled & $\mathbf{0 . 0 0 0 1}$ & $\mathbf{0 . 0 0 0 1}$ & & 0.0779 & $\mathbf{0 . 0 0 0 1}$ \\
\hline
\end{tabular}

Bold font denotes significant effects.

For the non-scaled data, significance maps for the effect of diagnosis (S7) and relative volume maps (S8) are included as Supplementary Data.

genders, compared to controls of the same gender(s). When no overall scaling is applied, regions including Crus II, VIIA and VIIB, exhibit statistics denoting excess tissue volume among FraX subjects. The significant deficit regions in the cerebellum remain consistent both with and without overall adjustment for brain scale (Figure S7).

Finally, there is a need to understand any effects of overall brain scale when reporting maps for the cerebellum, as with the cerebrum; in other words, it is informative to report results with and without adjustments for overall cerebral scale. To be consistent with our methods for describing cerebral differences, we report cerebellar results adjusted for overall cerebral scale, i.e., after global scaling of the cerebrum to the Colin 27 brain template. The Supplementary Data also reports cerebellar results without adjustment for overall cerebral scale, for completeness. In summary, we find morphometric differences in the cerebellum that survive corrections for multiple comparisons via permutation testing. After global scaling, the data show only significant tissue deficits in FraX (for both genders). If scaling is not applied, the unscaled data show both significant deficits and significant excesses in FraX. This is not an artifact, as the permutation tests show that both excess and deficits are significant after appropriate multiple comparisons corrections. Because effects of scaling are relevant, we summarize the significance of these findings in Table 2.

\section{Correlations with FMRP protein}

We also found that greater gray matter enlargement and ventricular expansion were significantly correlated with reduction in the Fragile X mental retardation protein (FMRP). In the FraX group, correlations between the Jacobian maps, which indicate local volumes, and FMRP levels were mapped and $r$-values (computed at each voxel via Spearman's rank correlation) and their corresponding significance levels for each gender are shown in Fig. 9. As predicted, females with FraX showed significant correlation between anatomy and FMRP levels but males did not. Unlike males with FraX (Fig. 9d), females (Figs. 9e and f) showed positive correlations in the anterior cingulate gyrus $(p=0.0026$; permutation test) and negative correlations in lateral ventricle area $(p=0.042$; permutation test $)$. In other words, the lower the FMRP level (low values being more atypical), the lower the Jacobian determinants in the cingulate gyrus, and the greater the expansion in the lateral ventricles. Prior fMRI studies (Menon et al., 2004) have associated reductions in fronto-striatal activation with protein reduction in FraX. In Fig. 9f, there is a region of protein-associated tissue reduction that appears to follow the anatomy of the anterior cingulate. While the ventricular correlation was hypothesized in advance and is borderline significant $(p=0.042)$, the cingulate effect was not hypothesized in advance. Although it survives multiple comparisons correction it should be regarded as a post hoc

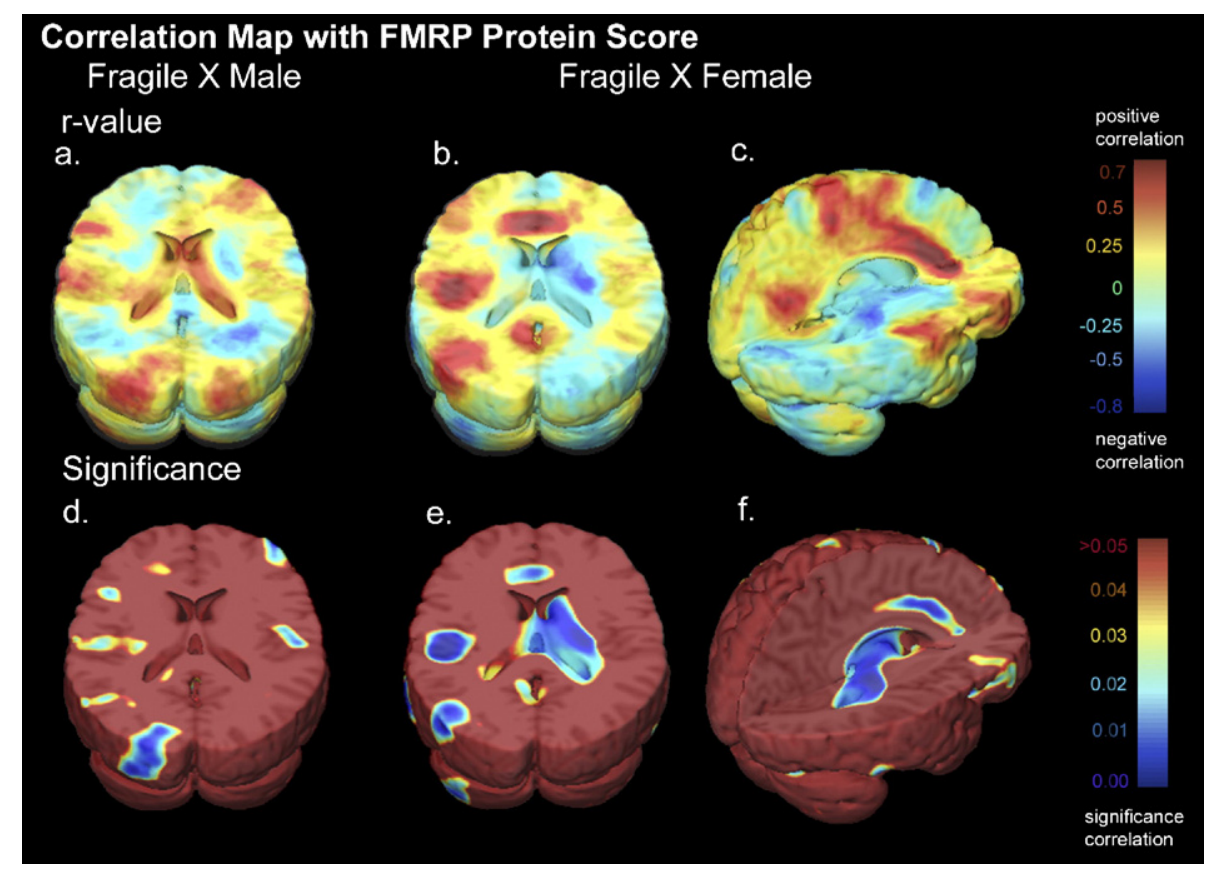

Fig. 9. FMRP protein correlations in males and females with FraX. (a-c), $r$-values that correlate FMRP protein score with tissue volumes are calculated and mapped to show the direction of the correlation (Spearman's rank correlation was used to avoid parametric assumptions). Their significance is shown in panels $\mathrm{d}-\mathrm{f}$ (blue colors show correlations that are significant at the uncorrected, voxel level). In the $r$-maps (a-c), red colors indicate regions that show volume reduction with reduction in FMRP protein, whereas blue colors indicate greater volume excesses with FMRP protein reduction. 
Table 3

Means and standard deviations (S.D.) for IQ scores in each subgroup: male controls, female controls, male and female controls, male FraX, female FraX, male and female FraX subjects pooled

\begin{tabular}{|c|c|c|c|c|c|c|c|}
\hline & & \multicolumn{3}{|l|}{ Mean } & \multicolumn{3}{|l|}{ S.D. } \\
\hline & & PIQ & FSIQ & VIQ & PIQ & FSIQ & VIQ \\
\hline \multirow{3}{*}{$\mathrm{CTL}$} & Male & 113.6 & 115.6 & 115.1 & 17.7 & 13.5 & 10.8 \\
\hline & Female & 110.4 & 112.4 & 112.7 & 10.2 & 9.4 & 10.1 \\
\hline & Both genders & 111.9 & 113.9 & 113.8 & 14.0 & 11.4 & 10.3 \\
\hline \multirow[t]{3}{*}{ FraX } & Male & 60.2 & 56.3 & 58.5 & 12.7 & 12.3 & 12.5 \\
\hline & Female & 84.6 & 82.4 & 82.9 & 24.2 & 23.5 & 21.0 \\
\hline & Both genders & 72.4 & 69.3 & 70.7 & 22.7 & 22.8 & 21.1 \\
\hline
\end{tabular}

FSIQ: full scale IQ, VIQ: verbal IQ, PIQ: performance IQ.

finding and should be confirmed in independent samples. Taken together, these findings suggest that the FMRI gene mutation leads to detectable changes in neuroanatomy that may be directly related to the molecular basis of FraX syndrome.

Supplementary Figure S6 shows the profile of correlations between FMRP levels and morphometric differences in the cerebellum of female and male subjects with FraX. Similar to the analysis for the cerebrum (Fig. 9), females with FraX (Figure $\mathrm{S} 6, \mathrm{~b})$ show some voxels, in the vermis region, where positive correlations were detected between FMRP and regional cerebellar volumes. In other words, the higher the FMRP level, the higher the Jacobian determinants in the cerebellar vermis. However, none of these effects was significant when corrected for multiple comparisons, using a search region encompassing the whole cerebellum. An alternative interpretation is possible if a smaller search region were used, because in prior studies, the posterior vermis size has been found to be smaller in FraX than in matched control subjects; for that reason the vermis might qualify as an a priori search region (Mostofsky et al., 1998). To control for type I error, however, we adopted the more conservative interpretation and suggest that no correlations between FMRP level and regional morphometry are detected here for the cerebellum, at least in this study.

\section{Correlations with IQ scores}

We correlated global and subdomain IQ measures - full scale IQ, verbal IQ, and performance IQ (summary statistics are reported in Table 3) - with morphometric differences detected within each gender and diagnostic group: normal males, normal females, male FraX and female FraX. The maps are paneled in Figure S9. The corrected significance values from the corresponding permutation tests are listed in Table 4 below. Significant positive correlations were detected only in FraX females. Correlations with FSIQ were followed up using post hoc tests of correlations with VIQ and PIQ, and these were also significant. As shown in Figure S9, d-f, some parietal regions in female FraX subjects show positive correlations with IQ scores. The Spearman's rank correlation test based on permutation, using the whole brain mask as a search region, does not show significant effects for any other group. Tests based on hypotheses that there would be positive correlations were conducted in four groups, so the Bonferroni-corrected significance for the FraX female group's correlation with FSIQ is $p=0.04(p=0.01$, if uncorrected for multiple groups tested). These results require replication before they can be interpreted strongly (Figs. 10 and 11).

\section{Discussion}

This study is the first to visualize the impact of Fragile $\mathrm{X}$ syndrome on brain structure in 3-dimensional detail. There were three main findings. First, consistent with prior studies using traditional volume measures (Eliez et al., 2001) we found marked enlargement in the periventricular structures, including the caudate. These effects were localized and mapped automatically, and were replicated in males and females, without detecting any sex differences (which were anticipated). Second, ventricular expansion was highly significant in the FraX group, and was linked with systemic reduction of the protein, FMRP, the production of which is suppressed or silenced in the disease. This linkage was in the expected direction, and was found in females but not males-as expected for an X-linked disorder, in that women exhibit a greater range of variation in disease burden and cognitive impairment due to $\mathrm{X}$ chromosome inactivation. A similar link between anterior cingulate tissue volumes was found, with correlation values as high as $r=0.5$ locally. This was not hypothesized in advance, and while it requires confirmation, cingulate deficits suggest a link between limbic system integrity and the severity of FMRP reduction in women. It is not clear if these expansions and reductions in specific tissue subtypes are immediate consequences of the protein deficiency, or whether they occur later in development as an adaptive response to the genetic mutation. Longitudinal studies are required to answer this question. White matter findings were mixed, showing only trend-level increases for white matter volumes (around $2-3 \%$ excess on average) in the parietal and temporal lobes. More detailed mapping of white matter volume differences with TBM suggested that some periventricular white matter regions were selectively increased in volume, by as much as $10 \%$ locally, but the white matter closer to the cortex (U-fibers rather than subcortical tracts and radiating white matter) was not significantly enlarged. This situation is analogous, at least superficially, to that seen in a recent autism study (Herbert et al., 2002), which found that autistic subjects showed significant enlargement in subcortical white matter regions but reductions in cortical (i.e., gyral) white matter. In autism, a theory has been advanced that, at least in some cases, there is excessive white matter growth in early

Table 4

Corrected $p$-values for the IQ scores correlated with morphometric differences in the Jacobian maps, in a search region defined by the whole brain mask

\begin{tabular}{|c|c|c|c|c|c|c|c|}
\hline & & \multicolumn{2}{|l|}{ FSIQ } & \multicolumn{2}{|l|}{ VIQ } & \multicolumn{2}{|l|}{ PIQ } \\
\hline & & Negative correlation & Positive correlation & Negative correlation & Positive correlation & Negative correlation & Positive correlation \\
\hline \multirow[t]{3}{*}{ Fragile $\mathrm{X}$} & Male & 0.241 & 0.974 & 0.170 & 0.960 & 0.112 & 0.673 \\
\hline & Female & 0.114 & 0.01 & 0.381 & 0.0133 & 0.0397 & 0.0118 \\
\hline & Both genders & 0.486 & 0.982 & 0.147 & 0.956 & 0.615 & 0.990 \\
\hline
\end{tabular}

Significant values are indicated in italics. 


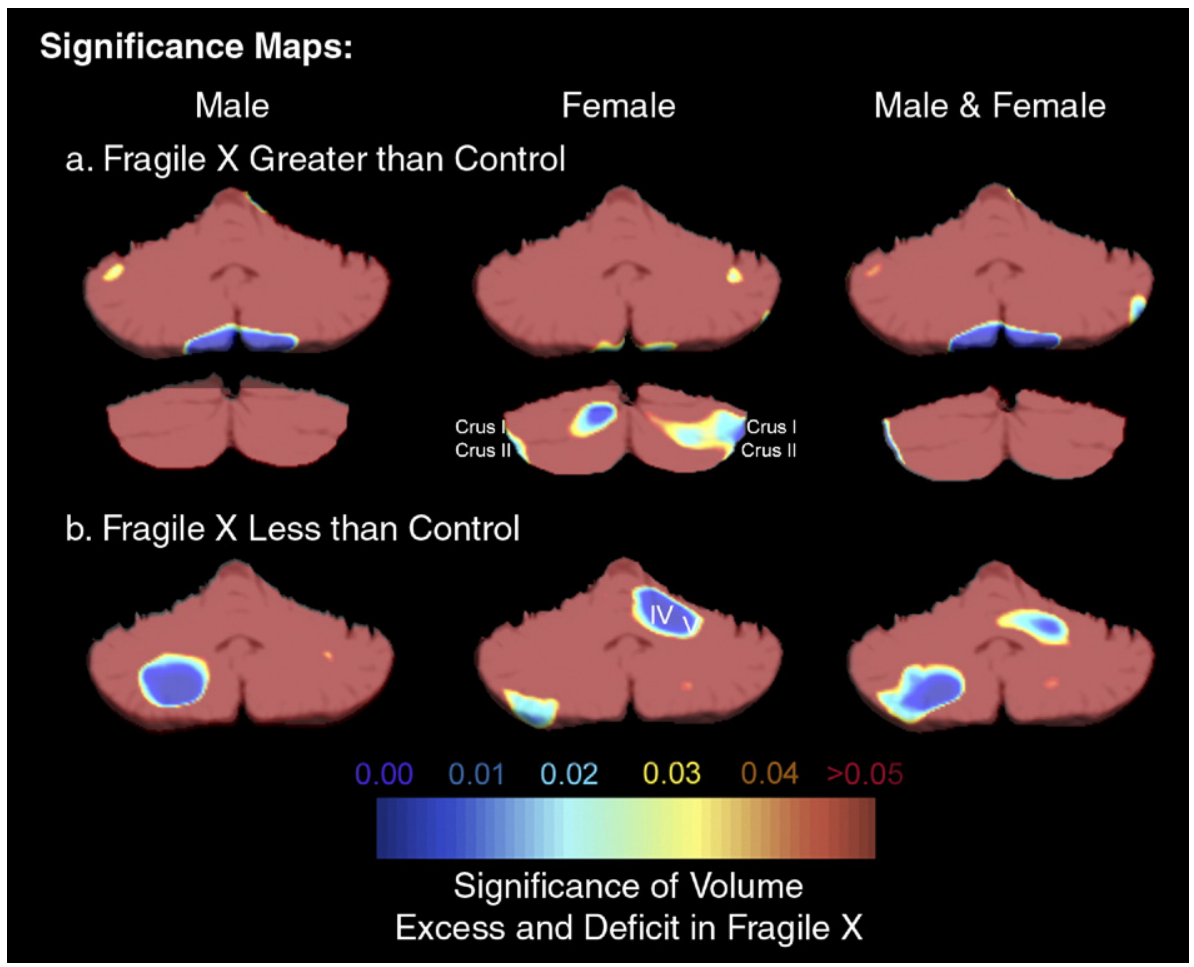

Fig. 10. Significance maps show group differences in regional cerebellar volumes between FraX subjects and controls. Significant volume differences in FraX subjects versus healthy controls are shown for males (left panels), females (middle) and for both genders pooled (right). First two rows (a) show excess volumes in FraX and the third row (b) shows significant deficits in FraX. Overall, there is a significant volume deficit in FraX (blue colors in b). The posterior cerebellar lobule of males with FraX exhibits reduced volume (b, left panel, indicated in blue colors); this effect is not detected in females. Females show evidence for reduced cerebellar tissue in lobules V and VI (blue colors in b, middle panel), compared to controls. When genders are pooled, lobules V and VI and the posterior lobes exhibit significant deficits (rightmost panel, in b).

childhood that normalizes somewhat by adulthood; these findings may be associated with increased brain size and head size in some persons with autism (Courchesne et al., 2003). In FraX, however, it is more common to associate developmental delay with reduced or delayed gray matter pruning, as these cellular substrates have been confirmed histologically. Other deficits in white matter, if

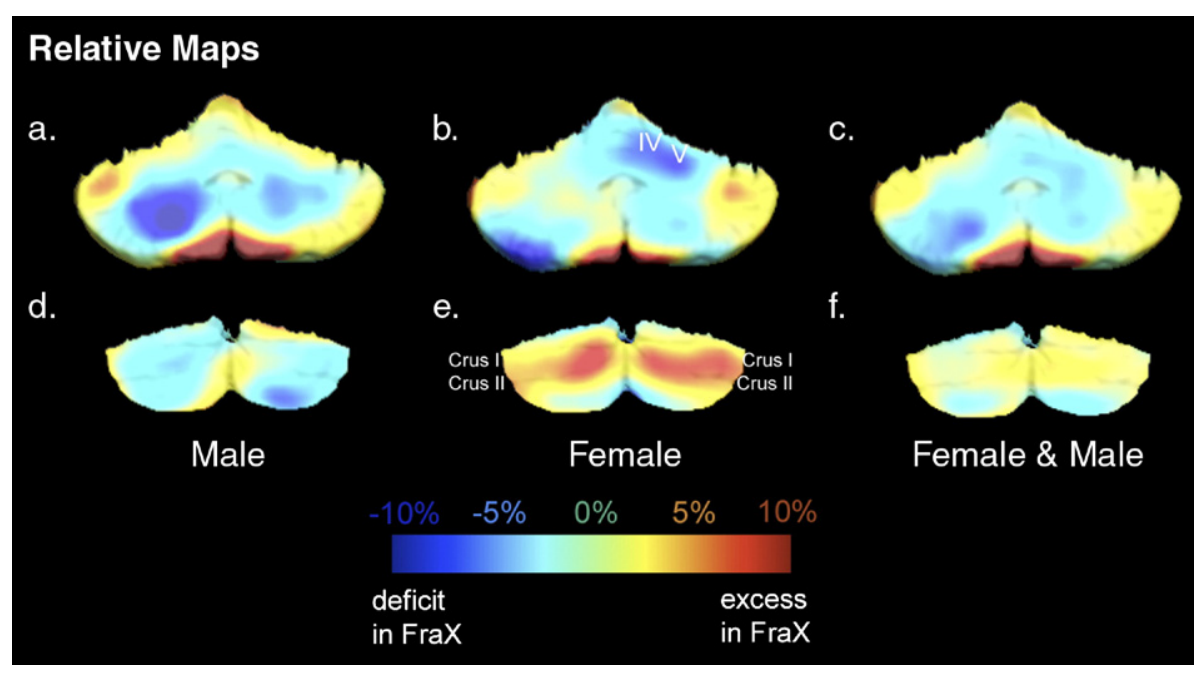

Fig. 11. Relative cerebellar tissue volume maps for FraX and control groups. Shown here are a coronal slice of the cerebellum in males (a, d), in females (b, e), and in males and females pooled (c, f). Slices were selected that contained regions significant for group differences at the voxel level, as in Fig. 10. The volume of the posterior lobule among subjects with FraX is less than controls in males (a), while there is no evidence for this difference in females (b). Cerebellar lobules $\mathrm{V}$ and VI show deficits among females with FraX (b) while the Crus I and II regions show relative expansion (e). When both genders are pooled, the maps show deficits in the posterior lobe overall (by around 5\% on average, in the FraX group compared to controls (c) and expansion in Crus I and II (with around 5\% more tissue in the FraX group; (f)). 
confirmed, would likely be interpreted as secondary disturbances in myelination or fiber migration that may be associated with the dendritic and neuronal abnormalities caused by the mutation. FMRP is not found to any significant degree in the white matter, or within axons or glial cells, making these white matter anomalies an interesting target for future study.

Longitudinal mapping of brain growth (e.g., Thompson et al., 2000, Chung et al., 2003) may be complemented in both autistic and FraX samples by diffusion tensor imaging or MR relaxometry, which may offer more sensitive indices of fiber integrity (Hendry et al., 2005). In a pilot DTI study of 10 females with FraX (BarneaGoraly et al., 2003), fronto-striatal circuits showed small areas of significantly reduced fractional anisotropy. Lowered FA is associated with aberrant myelination or myelin deterioration in a broad spectrum of degenerative and neurodevelopmental illnesses, and is often associated with cognitive impairment. Joint mapping of brain structure with TBM and DTI may be beneficial in future to determine whether fiber integrity and white matter excess are associated in FraX subjects, and whether these white matter alterations are found selectively in fronto-striatal regions or in deep white matter preferentially.

There were also two surprising negative findings. We expected disease effects to be greater in affected males versus females, but we found no gender-by-disease interactions in either the maps or volumes. While this negative result may reflect a lack of power in the design, it is somewhat paradoxical given the strong disease effects found in both males and females analyzed separately. In that respect, the maps of disease effects in males and females can be viewed as corroborating each other more than pointing to sex differences, which may be subtle or easier to identify with other imaging methods.

We also expected some benefit from measuring TBM effects using geometric means, as geometric or Riemannian means can correctly compute averages and differences in non-Euclidean tensor-valued data (see Woods, 2003; Pennec et al., 2004). Maps computed with geometric means essentially replicated the findings obtained with standard scalar statistics, and they did not offer greater statistical power. This contrasts with our findings in a recent TBM study of HIV/AIDS, where Lie group methods were superior to standard statistics for detecting disease-related brain atrophy (Lepore et al., 2006), both in the effect size and spatial extent of the detected effects. Any possible advantage of Lie group methods also depends to some degree on whether the brain shape differences are isotropic (i.e., tissue gain occurs in all directions equally) or whether the tissue shape changes have preferred directions, i.e., systematic tissue changes are more pronounced along certain directions. These biases can be identified using eigenanalysis of the Hencky strain tensors in TBM (Lepore et al., 2006).

\section{Gender differences}

We did expect to find gender differences in the magnitude and anatomical extent of the disease effect, so the fact that we did not is surprising. To better understand if this is a real effect, we computed geometrical mean anatomical templates (minimal deformation templates) for the diagnostic groups broken down by gender. The MDT technique provides one convenient method to compute a template with the average geometry for a group of subjects, so it can be used to make mean templates for each subgroup that can be presented for visual inspection. This offers some advantages over examining individual images as the mean geometry of each subgroup is visualized. The point of this analysis is that if any disease related differences are substantially more marked in men than women, this gender difference might be visually apparent in the mean geometric templates constructed for each group separately.

To allow a visual comparison between male and female mean anatomical templates, we subtracted the male MDT image from the female MDT image for each diagnosis and we also subtracted FraX from control. The images are included as Supplementary Figures S4 and S5. Corroborating the statistical findings reported in the statistical maps of group differences, the difference between the male FraX MDT and the male control MDT is indeed smaller than the difference between the MDTs constructed based on female FraX subjects and female controls. These images provide confidence that the results are reporting a real finding of an effect that is not greater in males than in females, rather than an artifactual effect. It is not legitimate to make statistical inferences based on these subtracted images (Bookstein, 2001), as in this case they are deliberately not registered with one another so that the mean geometries for each subgroup can be examined. In the main study, all individual images were nonlinearly registered to the same MDT template constructed from all subjects in the study, so voxel-wise comparisons are legitimate in that setting of fully registered images. Prior to the subtraction of the mean geometric templates, we first adjusted the mean intensities of each subject to be identical to avoid confounding effects of the different intensity ranges in each scan.

There are other possible reasons for the absence of a gender effect. The first logical possibility is that statistical power is always limited in a relatively small sample, so that the main effects are often detectable but second-order interactions are less reliably estimated or may go undetected. This argument is not entirely convincing as gender interactions were seen in other studies with comparable sample sizes (Eliez et al., 2001). A second logical possibility is that variance in the underlying signal may be higher in males, but Fig. 8 suggests that is not the case. Even though there are some regions in which variance is higher at the voxel level in the male group (e.g., the frontal poles in male controls), this is not found in regions with significant differences in FraX. Future comparisons of TBM versus volumetric methods in large samples of subjects should help to clarify whether the methods are differentially sensitive to the hypothesized interactions between gender and disease effects.

\section{Effects from signal inhomogeneity}

In a previous paper (Leow et al., 2006), we reported on the stability of candidate MR imaging sequences for tracking longitudinal brain change in the Alzheimer's Disease Neuroimaging Initiative (ADNI). We examined the effects of signal inhomogeneity due to the non-uniform RF inhomogeneity profiles of some receiver coils. In that study there was a substantial improvement in stability after intensity inhomogeneity correction with the program $\mathrm{N} 3$, in the sense that changes of lesser magnitude were recovered after intensity inhomogeneity correction, in a design where minimal change was expected (a longitudinal study with the repeat scan occurring after only 2 weeks).

To determine how any pre-existing inhomogeneities in the images might have affected the results in this FraX study, in supplementary Figures S1-S3, we compared results computed from intensity inhomogeneity-corrected images with the same maps computed from the uncorrected MR images. We confirmed 
that the effect of RF inhomogeneity correction was minimal, at least in this study. This finding is not really in disagreement with the finding in the ADNI study, as the boundary shifts induced by a smoothly varying bias field are typically less than a voxel, and certainly not likely to be systematically found in one group versus another. So any such net effect is small compared with the magnitude of the deformation fields used to compute expansion and contraction maps in this cross-sectional study. The effects of inhomogeneity correction are more appreciable in a longitudinal study, especially when the only signal detectable in the images is known to be small, such as the subvoxel changes seen in the 2-week longitudinal part of the ADNI study (Leow et al., 2006).

\section{Advantages and limitations of TBM}

TBM offers some advantages over traditional methods, as it visualizes the profile of structural deficits in the brain without timeconsuming specification of regions-of-interest. While this is the first study to use tensor-based morphometry to study FraX, TBM has proved to be useful for mapping brain structure differences in childhood-onset schizophrenia ( $\mathrm{Lu}$ et al., submitted for publication), bipolar illness (Foland et al., submitted for publication), normal brain development (Thompson et al., 2000; Chung et al., 2003; Hua et al., 2005), HIV/AIDS (Chiang et al., 2006, in press), and in twins (Lepore et al., 2006). We have performed several longitudinal studies mapping brain changes over time in individual subjects with semantic dementia (Leow et al., 2005, 2006, submitted for publication; see Studholme et al., 2001, for related work), and in subjects scanned before and after lithium treatment (Leow et al., 2006).

TBM can be performed with any nonlinear image registration approach, but there are some differences among registration approaches that affect their suitability for gauging differences in brain morphology. Our formulation maximizes mutual information between two images using a 3D elastic deformation that deforms one image to match another (Leow et al., 2005, 2006, submitted for publication), and is highly automated. Secondly, the computed deformation mappings are always guaranteed to be inverse-consistent, i.e., the same structures are matched if order of the input images is reversed (this property is desirable but not guaranteed by most commonly-used image registration algorithms). A minor weakness with TBM is that it is based on matching structures with similar intensity patterns, and may have limited success in matching cortical structures, which are extremely variable in patterning across subjects. As such, surface-based methods that can assess alterations in cortical gray matter thickness (Fischl et al., 2002; Salat et al., 2004; Thompson et al., 2004; Chung et al., 2004), and compensate for gyral variation across subjects by using large networks of sulcal features as landmarks for data alignment (Thompson et al., 2004), may be more powerful for detecting subtle alterations in the cortex, such as cortical thickening or cortical gray matter changes during development (Sowell et al., 2003; Gogtay et al., 2004; Shaw et al., 2006).

The time-course of the anatomical differences observed here will be assessed in the future with a longitudinal design, to help establish when the abnormalities emerge, and whether they tend to normalize or become more exaggerated in adulthood. TBM is especially powerful when applied in a longitudinal setting, as it can map growth profiles in individual children (Thompson et al., 2000), and can localize subtle group differences in growth rates of the order of $1-2 \%$ per year and over relatively short time intervals
(Chung et al., 2004; Leow et al., 2005; Lu et al., submitted for publication). Once the developmental trajectory of these structural brain changes is better established, the anatomy of FraX and its cellular correlates will be more completely understood.

\section{Acknowledgments}

This research was supported by grants from the National Institute for Biomedical Imaging and Bioengineering, the National Center for Research Resources, the National Institute on Aging, and the National Library of Medicine (EB01651, RR019771, AG016570, LM05639, to PMT) and grants from the National Institute of Mental Health (MH64708 and MH50047, to ALR).

\section{Appendix A. Supplementary data}

Supplementary data associated with this article can be found, in the online version, at doi:10.1016/j.neuroimage.2006.09.043.

\section{References}

Arsigny, V., Fillard, P., Pennec, X., Ayache, N., 2005. Fast and simple calculus on tensors in the Log-Euclidean framework. Proceedings of the 8th Int. Conf. on Medical Image Computing and Computer-Assisted Intervention-MICCAI 2005.

Ashburner, J., Friston, K.J., 1999. Nonlinear spatial normalization using basis functions. Hum. Brain Mapp. 7 (4), 254-266.

Ashburner, J., Friston, K.J., 2000. Voxel-based morphometry-The methods. NeuroImage 11 (6), 805-821.

Ashburner, J., Hutton, C., Frackowiak, R., Johnsrude, I., Price, C., Friston, K., 1998. Identifying global anatomical differences: deformation-based morphometry. Hum. Brain Mapp. 7 (5-6), 348-357.

Avants, B., Gee, J.C., 2004. Geodesic estimation for large deformation anatomical shape averaging and interpolation. NeuroImage 23 (S1), 39-50.

Avants, B.B., Schoenemann, P.T., Gee, J.C., 2006. Lagrangian frame diffeomorphic image registration: Morphometric comparison of human and chimpanzee cortex. Med. Image Anal. 10 (3), 397-412.

Barnea-Goraly, N., Eliez, S., Hedeus, M., Menon, V., White, C.D., Moseley, M., Reiss, A.L., 2003. White matter tract alterations in Fragile X syndrome: preliminary evidence from diffusion tensor imaging. Am. J. Med. Genet.: Neuropsychiatr. Genet. 118 (1), 81-88.

Beckel-Mitchener, A., Greenough, W.T., 2004. Correlates across the structural, functional, and molecular phenotypes of Fragile X syndrome. Ment. Retard. Dev. Disabil. Res. Rev. 10 (1), 53-59.

Bookstein, F.L., 2001. "Voxel-Based morphometry" should not be used with imperfectly registered images. NeuroImage 14, 1452-1462.

Cachier, P., Rey, D., 2000. Symmetrization of the non-rigid registration problem using inversion-invariant energies: application to multiple sclerosis. Med. Image Comput. Comput.-Assist. Interv.-MICCAI 472-481.

Chiang, M.C., Dutton, R.A., Hayashi, K.M., Toga, A.W., Lopez, O.L., Aizenstein, H.J., Becker, J.T., Thompson, P.M., 2006. Fluid registration of medical images using Jensen-Renyi divergence reveals 3D profile of brain atrophy in HIV/AIDS. IEEE Int. Symp. Biomed. Imag.

Chiang, M.C., Dutton, R.A., Hayashi, K.M., Lopez, O.L., Aizenstein, H.J, Toga, A.W., Becker, J.T., Thompson, P.M., in press. 3D pattern of brain atrophy in HIV/AIDS visualized using tensor-based morphometry. NeuroImage. [Electronic publication ahead of print].

Chung, M.K., Worsley, K.J., Paus, T., Cherif, C., Collins, D.L., Giedd, J.N., Rapoport, J.L., Evans, A.C., 2001. A unified statistical approach to deformation-based morphometry. NeuroImage 14, 595-606. 
Chung, M.K., Worsley, K.J., Robbins, S., Paus, T., Taylor, J., Giedd, J.N., Rapoport, J.L., Evans, A.C., 2003. Deformation-based surface morphometry applied to gray matter deformation. NeuroImage 18 (2), 198-213.

Chung, M.K., Dalton, K.M., Alexander, A.L., Davidson, R.J., 2004. Less white matter concentration in autism: $2 \mathrm{D}$ voxel-based morphometry. NeuroImage 23 (1), 242-251.

Courchesne, E., Carper, R., Akshoomoff, N., 2003. Evidence of brain overgrowth in the first year of life in autism. JAMA 290 (3), $337-344$.

Davatzikos, C., Vaillant, M., Resnick, S.M., Prince, J.L., Letovsky, S., Bryan, R.N., 1996. A computerized approach for morphological analysis of the corpus callosum. J. Comput. Assist. Tomogr. 20 (1), 88-97.

Davatzikos, C., Genc, A., Xu, D., Resnick, S.M., 2001. Voxel-based morphometry using the RAVENS maps: methods and validation using simulated longitudinal atrophy. NeuroImage 14 (6), 1361-1369.

de Vries, B.B., van den Ouweland, A.M., Mohkamsing, S., Duivenvoorden, H.J., Mol, E., Gelsema, K., van Rijn, M., Halley, D.J., Oostra, L.A., Tibben, B.A., Niermeijer, A., 1997. Screening and diagnosis for the Fragile $\mathrm{X}$ syndrome among the mentally retarded: an epidemiological and psychological survey. Am. J. Hum. Genet. 61 (3), 660-667.

Eliez, S., Blasey, C.M, Freund, L.S., Hastie, T., Reiss, A., 2001. Brain anatomy, gender, and IQ in children and adolescents with Fragile X syndrome. Brain 124, 1610-1618.

Fischl, B., Salat, D.H., Busa, E., Albert, M., Dieterich, M., Haselgrove, C., van der Kouwe, A., Killiany, R., Kennedy, D., Klaveness, S., Montillo, A., Makris, N., Rosen, B., Dale, A.M., 2002. Whole brain segmentation: automated labeling of neuroanatomical structures in the human brain. Neuron 33 (3), 341-355.

Foland, L.C., Altshuler, L.L., Leow, A.D., Lee, A.D., Lu, A., Asuncion, D., Toga, A.W., Thompson, P.M., (submitted for publication). A TensorBased Morphometric Study of Bipolar Disorder.

Fox, N.C., Freeborough, P.A., Rossor, M.N., 1996. Visualisation and quantification of rates of atrophy in Alzheimer's disease. Lancet 348 (9020), 94-97.

Fox, N.C., Crum, W.R., Scahill, R.I., Stevens, J.M., Janssen, J.C., Rossor, M.N., 2001. Imaging of onset and progression of Alzheimer's disease with voxel-compression mapping of serial magnetic resonance images. Lancet 358 (9277), 201-205.

Galvez, R., Greenough, W.T., 2005. Sequence of abnormal dendritic spine development in primary somatosensory cortex of a mouse model of the Fragile X mental retardation syndrome. Am. J. Med. Genet. A 135 (2), $155-160$.

Giedd, J.N., Snell, J.W., Lange, N., Rajapakse, J.C., Casey, B.J., Kozuch, P.L., 1996. Quantitative magnetic resonance imaging of human brain development: ages 4-18. Cereb. Cortex 6, 551-560.

Giedd, J.N., Blumenthal, J., Jeffries, N.O., Castellanos, F.X., Liu, H., Zijdenbos, A., Paus, T., Evans, A.C., Rapoport, J.L., 1999. Brain development during childhood and adolescence: a longitudinal MRI study. Nat. Neurosci. 2, 861-863.

Gogtay, N., Giedd, J., Lusk, L., Hayashi, K.M., Greenstein, D.K., Vaituzis, A.C., Nugent, T.F., Herman, D., Clasen, L.S., Toga, A.W., Rapoport, J.L., Thompson, P.M., 2004. Dynamic mapping of human cortical development during childhood through early adulthood. Proc. Natl. Acad. Sci. U. S. A. 101, 8174-8179.

Gustavson, K.H., Blomquist, H.K., Holmgren, G., 1986. Prevalence of the Fragile-X syndrome in mentally retarded boys in a Swedish county. Am. J. Med. Genet. 23, 581-587.

Hendry, J., DeVito, T., Gelman, N., Densmore, M., Rajakumar, N., Pavlosky, W., Williamson, P., Thompson, P.M., Drost, D., Nicolson, R., 2005. White Matter Abnormalities in Autism Detected Through Transverse Relaxation Time Imaging. NeuroImage Oct. 5; [Electronic publication ahead of print].

Herbert, M.R., Harris, G.J., Adrien, K.T., Ziegler, D.A., Makris, N., Kennedy, D.N., Lange, N.T., Chabris, C.F., Bakardjiev, A., Hodgson, J., Takeoka, M., Tager-Flusberg, H., Caviness Jr., V.S., 2002. Abnormal asymmetry in language association cortex in autism. Ann. Neurol. 52 (5), 588-596.
Hinton, V.J., Brown, W.T., Wisniewski, K., Rudelli, R.D., 2005. Analysis of neocortex in three males with the Fragile X syndrome. Am. J. Med. Genet. vol. 41 (3), P289-P294.

Hodapp, R.M., Dykens, E.M., Hagerman, R., Schreiner, R., Lachiewicz, A., Leckman, J., 1990. Developmental implications of changing IQ trajectories of males with Fragile X syndrome. J. Am. Acad. Child Adolesc. Psych. 29, 214-219.

Holmes, C.J., Hoge, R., Collins, L., Woods, R., Toga, A.W., Evans, A.C., 1998. Enhancement of MR images using registration for signal averaging. J. Comput. Assist. Tomogr. 22, 324-333.

Hua, X., Leow, A.D., Levitt, J.G., Thompson, P.M., Toga, A.W., 2005. Detecting Brain Growth Patterns in Normal Children using TensorBased Morphometry. 35th Annual Meeting of the Society for Neuroscience, Washington, DC.

Irwin, S.A., Galvez, R., Greenough, W.T., 2000. Dendritic spine structural anomalies in Fragile-X mental retardation syndrome. Cereb. Cortex 10 (10), 1038-1044

Jakala, P., Hanninen, T., Ryynanen, M., Laakso, M., Partanen, K., Mannermaa, A., 1997. Fragile-X: neuropsychological test performance, CGG triplet repeat lengths, and hippocampal volumes. J. Clin. Invest. 100, 331-338.

Kochunov, P., Lancaster, J., Thompson, P.M., Woods, R.P., Hardies, J., Fox, P.T., 2001. Regional spatial normalization: towards an optimal target. J. Comput. Assist. Tomogr. 25 (5), 805-816.

Kochunov, P., Lancaster, J., Thompson, P.M., Toga, A.W., Brewer, P., Hardies, J., Fox, P.T., 2002. An optimized individual target brain in the talairach coordinate system. NeuroImage 17 (2), 922-927.

Kochunov, P.K., Lancaster, J.L., Hardies, J., Thompson, P.M., Woods, R.P., Cody, J.D., Hale, D.E., Laird, A., Fox, P.T., 2005. Mapping structural differences of the corpus callosum in individuals with $18 \mathrm{q}$ deletions using targetless regional spatial normalization. Hum. Brain Mapp. 24 (4), 325-331.

Leow, A., Huang, S.C., Geng, A., Becker, J., Davis, S., Toga, A., Thompson, P., 2005. Inverse consistent mapping in $3 \mathrm{D}$ deformable image registration: its construction and statistical properties. IPMI2005, Lect. Notes Comput. Sci. 493-503.

Leow, A.D., et al., For the ADNI Preparatory Phase Study, 2006. Longitudinal Stability of MRI for Mapping Brain Change Using Tensor-Based Morphometry. NeuroImage Feb. 7; [Electronic publication ahead of print].

Leow, A.D., Lee, A.D., Klunder, A.D, Lu, A., Huang, S., Becker, J.T., Davis, S.W., Toga, A.W., Thompson, P.M., submitted for publication. Statistical properties of Jacobian map and inverse-consistent deformations in non-linear image registration.

Lepore, N., Brun, C.A., Chiang, M.C., Chou, Y.Y., Dutton, R.A., Hayashi, K.M., Becker, J.T., Aizenstein, H.J., Lopez, O.L., Toga, A.W., Thompson, P.M., 2006. Multivariate Statistics of the Jacobian Matrices in Tensor-Based Morphometry and their application to HIV/AIDS, $9^{\text {th }}$ IEEE Conference on Medical Image Computer and Computer Assisted Intervention (MICCAI2006).

Lu, A., Leow, A.D., Lee, A.D., Gogtay, N., Rapoport, J.L., Toga, A.W., Thompson, P.M., submitted for publication. Growth Pattern Abnormalities in Childhood-onset Schizophrenia Visualized using TensorBased Morphometry.

Menon, V., Leroux, J., White, C.D., Reiss, A.L., 2004. Frontostriatal deficits in Fragile X syndrome: relation to FMR1 gene expression. Proc. Natl. Acad. Sci. 101, 3615-3620.

Miller, M.I., 2004. Computational anatomy: shape, growth, and atrophy comparison via diffeomorphisms. NeuroImage 23 (Suppl. 1), S19-S33.

Mostofsky, S.H., Mazzocco, M.M., Aakalu, G., Warsofsky, I.S., Denckla, M.B., Reiss, A.L., 1998. Decreased cerebellar posterior vermis size in Fragile $\mathrm{X}$ syndrome: correlation with neurocognitive performance. Neurology 50 (1), 121-130.

Pennec, X., Fillard, P., Ayache, N., 2004. A Riemannian framework for tensor computing. Int. J. Comput. Vis. 66 (1), 41-66.

Pfefferbaum, A., Mathalon, D.H., Sullivan, E.V., Rawles, J.M., Zipursky, R.B., Lim, K.O., 1994. A quantitative magnetic resonance imaging 
study of changes in brain morphology from infancy to late adulthood. Arch. Neurol. 51, 974-987.

Reiss, A.L., Kazazian, H.H., Krebs, C.M., McAughan, A., Boehm, C.D., Abrams, M.T., Nelson, D.L., 1994. Frequency and stability of the Fragile X permutation. Hum. Mol. Genet. 3 (3), 393-398.

Reiss, A.L., Abrams, M.T., Singer, H.S., Ross, J.L., Denckla, M.B., 1996. A volumetric imaging study. Brain 119, 1763-1774.

Salat, D.H., Buckner, R.L., Snyder, A.Z., Greve, D.N., Desikan, R.S.R., Busa, E., Morris, J.C., Dale, A.M., Fischl, B., 2004. Thinning of the cerebral cortex in aging. Cereb. Cortex 14, 721-730.

Schapiro, M.B., Murphy, D.G., Hagerman, R.J., Azari, N.P., Alexander, G.E., Miezejeski, C.M., Hinton, V.J., Horwitz, B., Haxby, J.V., Kumar, A., 19954. Adult fragile X syndrome: neuropsychology, brain anatomy, and metabolism. Am. J. Med. Genet. 60 (6), 480-493.

Schmahmann, J.D., Doyon, J., Toga, A.W., Petrides, M., Evans, A.C., 2000. MRI Atlas of the Human Cerebellum. Academic Press, San Diego.

Shattuck, D.W., Leahy, R.M., 2002. Brainsuite: an automated cortical surface identification tool. Med. Image Anal. 8 (2), 129-142.

Shattuck, D.W., Sandor-Leahy, S.R., Schaper, K.A., Rottenberg, D.A., Leahy, R.M., 2001. Magnetic resonance image tissue classification using a partial volume model. NeuroImage 13, 856-876.

Shaw, P., Greenstein, D., Lerch, J., Clasen, L., Lenroot, R., Gogtay, N., Evans, A., Rapoport, J., Giedd, J., 2006. Intellectual ability and cortical development in children and adolescents. Nature 440 (30 March 2006).

Shen, D., Davatzikos, C., 2003. Very high-resolution morphometry using mass-preserving deformations and HAMMER elastic registration. NeuroImage 18 (1), 28-41.

Sled, J.G., Zijdenbos, A.P., Evans, A.C., 1998. A nonparametric method for automatic correction of intensity nonuniformity in MRI data. IEEE Trans. Med. Imag.. 17 (1), 87-97.

Sowell, E.R., Peterson, B.S., Thompson, P.M., Welcome, S.E., Henkenius, A.L., Toga, A.W, 2003. Mapping cortical change across the human life span. Nat. Neurosci. 6, 309-315.

Sowell, E.R., Thompson, P.M., Leonard, C.M., Welcome, S.E., Kan, E., Toga, A.W., 2004. Longitudinal mapping of cortical thickness and brain growth in normal children. J. Neurosci. 24 (38), 8223-8231.

Studholme, C., Cardenas, V., 2004. A template-free approach to volumetric spatial normalization of brain anatomy. Pattern Recogn. Lett. 25, $1191-1202$.

Studholme, C., Cardenas, V., Schuff, N., Rosen, H., Miller, B., Weiner, M., 2001. Detecting spatially consistent structural differences in Alzheimer's and fronto temporal dementia using deformation morphometry. Proc. Int Conf. Med. Image Comput. Comput. Assist. Interv. (MICCAI) 41-48.

Studholme, C., Cardenas, V., Maudsley, A., Weiner, M., 2003. An intensity consistent filtering approach to the analysis of deformation tensor derived maps of brain shape. NeuroImage 19 (4), 1638-1649.

Studholme, C., Cardenas, V., Blumenfeld, R., Schuff, N., Rosen, H.J., Miller, B., Weiner, M., 2004. Deformation tensor morphometry of semantic dementia with quantitative validation. NeuroImage 21 (4), 1387-1398.

Tamanini, F., Meijer, N., Verheij, C., Willems, P.J., Galjaard, H., Oostra, B.A., Hoogeveen, A.T., 1996. FMRP is associated to the ribosomes via RNA. Hum. Mol. Genet. 5, 809-813.

Teipel, S.J., Alexander, G.E., Schapiro, M.B., Moller, H.J., Rapoport, S.I., Hampel, H., 2004. Age-related cortical grey matter reductions in nondemented Down's syndrome adults determined by MRI with voxelbased morphometry. Brain 127 (Pt. 4), 811-824.

Thompson, P.M., Giedd, J.N., Woods, R.P., MacDonald, D., Evans, A.C., Toga, A.W., 2000. Growth patterns in the developing brain detected by using continuum mechanical tensor maps. Nature 404 (6774), 190-193.

Thompson, P.M., Hayashi, K.M., Simon, S.L., Geaga, J.A., Hong, M.S., Sui, Y., Lee, J.Y., Toga, A.W., Ling, W., London, E.D., 2004. Structural abnormalities in the brains of human subjects who use methamphetamine. J. Neurosci. 24 (26), 6028-6036.

Toga, A.W., Thompson, P.M., Sowell, E.R., 2006. Mapping Brain Maturation. Trends Neurosci. Feb 9; [Electronic publication ahead of print].

Twining, C.J., Cootes, T., Marsland, S., Petrovic, V., Schestowitz, R., Taylor, C.J., 2005. A unified information-theoretic approach to groupwise non-rigid registration and model building. Information Processing in Medical Imaging (IPMI) 2005, Glenwood Springs, Colorado, July 11-15.

Weiler, I.J., Greenough, W.T., 1993. Metabotropic glutamate receptors trigger postsynaptic protein synthesis. Proc. Natl. Acad. Sci. U. S. A. 90, $7168-7171$.

Willemsen, R., Oostra, B.A.;, Bassell, G.J., Dictenberg, J., 2004. The Fragile $\mathrm{X}$ syndrome: from molecular genetics to neurobiology. Ment. Retard. Dev. Disabil. Res. Rev. 10 (1), 60-67.

Wisniewski, K.E., Segan, S.M., Miezejeski, C.M., Sersen, E.A., Rudelli, R.D., 1991. The Fra(X) syndrome: neurological, electrophysiological, and neuropathological abnormalities. Am. J. Med. Genet. 38 (2-3), 476-480.

Woods, R.P., 2003. Characterizing volume and surface deformations in an atlas framework: theory, applications, and implementation. NeuroImage 18 (3), 769-788. 